\title{
Acromioclavicular joint acceleration-deceleration injury as a cause of persistent shoulder pain: Outcome after arthroscopic resection
}

\author{
Ehud Atoun, Artan-Athanasios Bano ${ }^{1}$, Alexander Van Tongel ${ }^{1}$, Ali Narvani ${ }^{1}$, Giuseppe Sforza ${ }^{l}$, Ofer Levy ${ }^{1}$
}

\begin{abstract}
Background: Shoulder pain in general and acromioclavicular joint (ACJ) pain specifically is common after acceleration-deceleration injury following road traffic accident (RTA). The outcome of surgical treatment in this condition is not described in the literature. The aim of the present study was to report the outcome of arthroscopic resection of the ACJ in these cases.

Materials and Methods: Nine patients with localized ACJ pain, resistant to nonoperative treatment were referred on an average 18 months after the injury. There were 3 male and 6 females. The right shoulder was involved in seven patients and the left in two. The average age was 38.9 years (range $29-46$ years). All presented with normal X-rays but with torn acromioclavicular joint disc and effusion on magnetic resonance imaging (MRI). Arthroscopic ACJ excision arthroplasty was performed in all patients.

Results: At a mean followup of 18 month, all patients had marked improvement. The Constant score improved from 36 to 81 , the pain score from 3/15 to $10 / 15$ and the patient satisfaction improved from 3.5/10 to 9.3/10.

Conclusion: Arthroscopic ACJ excision arthroplasty, gives good outcomes in patients not responding to conservative management in ACJ acceleration-deceleration injury.
\end{abstract}

Key words: Acceleration-deceleration injury, acromioclavicular joint, whiplash, arthroscopic resection

\section{INTRODUCTION}

$\mathrm{T}$ The term "whiplash injury" is used to describe the constellation of symptoms triggered by road traffic accident (RTA) with an acceleration-deceleration mechanism. The most common symptoms are neck pain and stiffness, occipital headache, thoracolumbar back pain and paresthesia in the upper limbs. ${ }^{1-5}$

Shoulder pain was previously described in patients after acceleration-deceleration injury. This pain was attributed to referred pain from the neck and to impingement syndrome

Department of Orthopaedic, Barzilai Medical Center, Ashkelon, Ben-Gurion University of the Negev, Beer Sheva, Israel, ${ }^{1}$ The Reading Shoulder Unit, Department of Orthopedics, Royal Berkshire Hospital, Reading, Berkshire, United Kingdom

Address for correspondence: Dr. Ehud Atoun,

Department of Orthopaedic, Barzilai Medical Center, Ashkelon, Israel.

E-mail: dratoun@gmail.com

\begin{tabular}{|l|l|}
\hline \multicolumn{2}{|c|}{ Access this article online } \\
\hline Quick Response Code: & Website: \\
\hline & www.ijoonline.com \\
\cline { 2 - 2 } & \\
\hline
\end{tabular}

of the shoulder due to an impaired glenohumeral and scapulothoracic rhythm. ${ }^{1,6,7}$

We identified in our practice, a group of patients with persistent shoulder pain after an acceleration-deceleration injury in RTA. All patients originally complained of shoulder and neck pain, but while their neck symptoms improved with time, the shoulder symptoms failed to improve despite treatment with analgesics, anti inflammatory drugs and physiotherapy. These patients complained of pain localized to the acromioclavicular joint (ACJ) with a no obvious pathology on X-rays.

The purpose of this study is to describe ACJ injury as a cause of persistent shoulder pain after an acceleration-deceleration RTA trauma and to assess the outcome of arthroscopic excision in this condition in cases who did not respond to conservative treatment.

\section{Materials and Methods}

We performed a retrospective audit search in the database of our Institute for patients who underwent ACJ excision arthroplasty from $1^{\text {st }}$ January to $31^{\text {st }}$ December 2009. Data collected included age, sex, side, preceding injury, sitting position in the car during the accident, presence or absence of seatbelt, duration of symptoms, previous 
treatments, clinical findings and tests (ACJ tenderness, cross-arm adduction test and ACJ pain relief after local anesthetic injection test), radiological findings, surgical findings and the Constant score. ${ }^{8}$

Our inclusion criteria were symptoms triggered by RTA, failure of nonoperative treatment for at least 6 months, localized tenderness at the $\mathrm{ACJ}$ and positive, but temporary response to a local anesthetic injection. Our exclusion criteria were marked arthritic changes at the ACJ on X-rays and history of shoulder region pain prior to the accident.

Constant scores and patient satisfaction scores were obtained in all patients preoperatively and at each followup. Patient satisfaction was determined by use of a visual analog scale ranging from 0 to 10 points, in increasing order of satisfaction.

\section{Results}

9 patients matched our criteria. Of these, 6 were female and 3 were male. All were involved in RTA with acceleration-deceleration insult. None of them had any neck or shoulder symptoms prior to the accident. Shoulder pain and symptoms did not appear immediately at the accident, but usually a few hours or a couple of days later. The average time between the accident and referral to us was 18 months (range 4-120 months) with majority of patients coming more than 12 months after the accident.

The average age was 38.9 years (range 29-46 years). Seven patients were drivers and two patients were front seat passengers. All the cars were right hand drives and all patients wore seat belts during RTA. In the seven drivers, the right shoulder was involved, whereas the two front seat passengers had symptoms in their left shoulder. The clinical presentation was typical, with the patients complaining of constant dull pain in the shoulder, usually on top, with some tenderness over the upper part of the trapezius muscle. They described the pain in the neck radiating to the shoulder (or vice versa) with intermittent acute episodes of sharp pain accompanied by "clicking" or "locking" sensation in the shoulder. These appeared during terminal elevation and abduction, sometimes on descent of the arm or in extreme rotations.

Clinical examination revealed localized tenderness over the ACJ with high arc pain and occasional sharp pain with loud clicking from the ACJ. There was positive cross body arm adduction test. All the patients in this group had temporary pain relief following injection to the ACJ with local anesthetic and steroids ( $1 \mathrm{ml} 0.25 \%$ Marcaine $+40 \mathrm{mg} / 1 \mathrm{ml}$ Depomedrone). This injection served as the diagnostic test.
$\mathrm{X}$-rays were overall unremarkable. No gross degenerative changes of the ACJ or signs of ACJ instability were seen in any of the patient. Magnetic resonance imaging (MRI) scans of the shoulder prior to surgery in four patients demonstrated pathological findings in the ACJ and an associated effusion [Figure 1].

Owing to the long standing failure of nonoperative treatment all patients underwent surgery in which an arthroscopic evaluation of the glenohumeral joint and the subacromial bursa including the ACJ was performed. During the procedure, positive subacromial impingement signs were found in five patients and a partial, bursal side tear that involved $<50 \%$ of the tendon width in one patient. After opening of the inferior capsule of the ACJ, a torn ACJ intraarticular disc was found in all the patients [Figure 2a and b]. In all patients arthroscopic ACJ excision arthroplasty was performed with resection of the remnants of the intraarticular disc and resection of $4 \mathrm{~mm}$ from each side of the joint, preserving the integrity of the superior capsule of the ACJ.

At a mean followup of 18 months (range 12-23 months) all patients had marked improvement in symptoms and pain, the Constant score, pain score and overall satisfaction. The mean Constant score improved from 36 points preoperatively (range 28-45) to 81 points (range 72-90). The pain score (visual analog scale from 0 to 15 a subscore of the Constant score) improved from $10 / 15$ to $3 / 15$ and the patient satisfaction improved from $3.5 / 10$ to $9.3 / 10$ in the last followup.

\section{Discussion}

Shoulder pain is common after acceleration-deceleration injury and can trouble the patient for a long time. This was previously attributed as referred pain from the neck or due

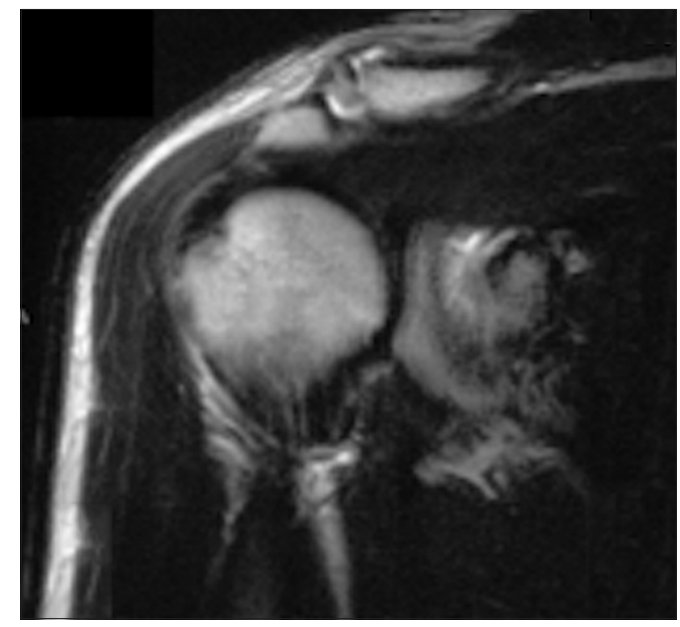

Figure 1: Magnetic resonance imaging scan showing a torn acromioclavicular joint intraarticular disc with effusion in the joint 
to an impingement syndrome of the shoulder following an impaired glenohumeral and scapulothoracic rhythm. ${ }^{1,6,7}$ Omar et al., ${ }^{9}$ reported on damage to the long thoracic and spinal accessory nerves as a cause of dysfunction of the trapezius muscle and possible abnormal scapulohumeral rhythm. Gorski and Schwartz ${ }^{10}$ reported shoulder pain in whiplash injuries localized to the supraspinatus muscle and called this condition as Referred Shoulder Impingement Syndrome.

The ACJ is a diarthrodial joint with a fibrocartilaginous disc that has been shown to involute with age and begin to degenerate by the age of 40 years. ${ }^{11,12}$ Previous preliminary studies suggested the ACJ as a possible cause of what was described as the "whiplash injury of the shoulder". ${ }^{13,14}$

The main goal of this study was to point out the possibility of the diagnosis of a frequently missed, occult ACJ injury during acceleration-deceleration trauma in RTA setting. In the case series, the ACJ symptoms appeared following an acceleration-deceleration trauma (Whiplash) in RTA, yet, the diagnosis was missed or delayed in these cases and wrongly related to the whiplash injury of the cervical spine.

Tossy et al., ${ }^{15}$ and Allman ${ }^{16}$ originally classified ACJ injuries into three grades. This classification was subsequently modified by Williams et al. ${ }^{17}$ into six types. These classifications mainly address the radiological deformity of the joint. Occult injuries to the ACJ may involve AC and coracoclavicular ligament injuries with Tossy et al. and Allman (or Rockwood) grade I and II ACJ separation. In these cases there is no clear evidence of instability of the $\mathrm{AC}$ joint, yet significant intraarticular ACJ injury is frequently observed.

Due to the absence of radiological changes or joint instability, occult injury to the ACJ including intraarticular disc damage is usually classified as a grade I injury in which a nonoperative treatment is recommended. Unfortunately these patients can demonstrate persistent pain, which is resistant to nonoperative treatment.

Heers et al. ${ }^{17,18}$ have demonstrated that MRI allows the differentiation between intraarticular fluid, disk tissue,

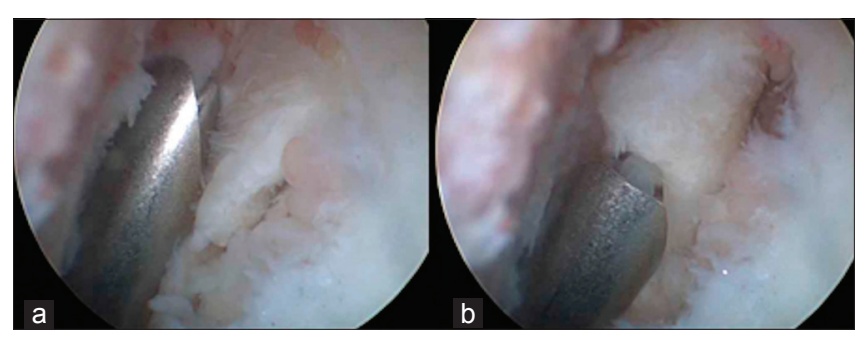

Figure 2: $(a$ and b) Arthroscopic view of a torn acromioclavicular joint intraarticular disc cortical bone and joint capsule. In our group of nine patients with persistent localized ACJ pain after whiplash injury, MRI scan was performed in four and demonstrated subtle intraarticular pathology and effusion [Figure 1]. Intraoperatively a torn intraarticular disc was found in all the patients [Figure $2 \mathrm{a}$ and $\mathrm{b}$ ]. We could not confirm whether the torn disc was a direct consequence of the injury or it was a degenerative disc that had further damaged and deteriorated following the accident. Nevertheless, all these patients became symptomatic following the accident. All of our patients had the seat belt stabilizing the distal clavicle of the injured shoulder while sustaining an acceleration-deceleration injury [Figure 3].

We have seen other patients presenting with similar clinical picture with persistent shoulder pain following an acceleration-deceleration injury in RTA, where conservative treatment with injection to the ACJ with local anesthetic and depomedrone have settled their pain, although some pain free occasional clicking persisted. The injection concluded the treatment in these cases.

Owing to the failure of the nonoperative treatment, the patients in our series underwent arthroscopic ACJ excision arthroplasty with resection of the disc. All were relieved of pain with marked improvement in their Constant scores, pain scores and satisfaction that continued to improve at an average of 18 months after surgery. Our findings suggest that the ACJ is prone to injury when subjected to acceleration-deceleration injury between stabilized distal clavicle and the mobile acromion.

Clinicians should be aware to the possibility of a frequently missed, occult injury to the ACJ and the intraarticular disc as a cause of continuing pain and disability following acceleration-deceleration trauma (Whiplash) in RTA. Focused clinical examination and MRI can assist in diagnosing this injury. A good diagnostic tool is intraarticular

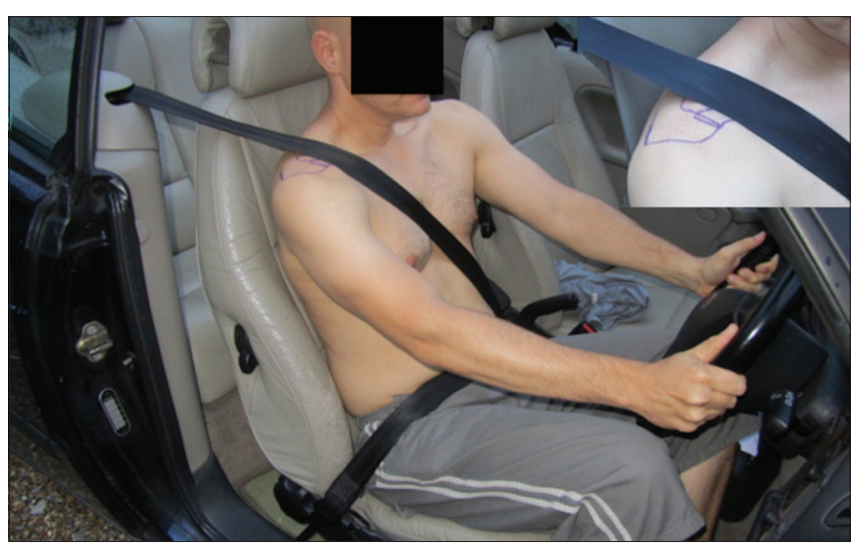

Figure 3: Clinical photograph showing the seatbelt positioning on the right shoulder of the driver, stabilizing the distal clavicle 
injection to the ACJ of local anesthetic and steroid. Surgery, if needed, with arthroscopic ACJ excision arthroplasty, produces good outcomes in patients unresponsive to nonoperative management.

\section{References}

1. Hildingsson C, Toolanen G. Outcome after soft-tissue injury of the cervical spine. A prospective study of 93 car-accident victims. Acta Orthop Scand 1990;61:357-9.

2. Hohl M. Soft-tissue injuries of the neck in automobile accidents. Factors influencing prognosis. J Bone Joint Surg Am 1974;56:1675-82.

3. Maimaris C, Barnes MR, Allen MJ. 'Whiplash injuries' of the neck: A retrospective study. Injury 1988;19:393-6.

4. Ratliff $\mathrm{AH}$. Whiplash injuries. J Bone Joint Surg $\mathrm{Br}$ 1997;79:517-9.

5. Spitzer WO, Skovron ML, Salmi LR, Cassidy JD, Duranceau J, Suissa S, et al. Scientific monograph of the Quebec task force on whiplash-associated disorders: Redefining "whiplash" and its management. Spine (Phila Pa 1976) 1995;20:1S-73.

6. Abbassian A, Giddins GE. Subacromial impingement in patients with whiplash injury to the cervical spine. J Orthop Surg Res 2008;3:25.

7. Chauhan SK, Peckham T, Turner R. Impingement syndrome associated with whiplash injury. J Bone Joint Surg $\mathrm{Br}$ 2003;85:408-10.

8. Ban I, Troelsen A, Christiansen DH, Svendsen SW, Kristensen MT. Standardised test protocol (Constant Score) for evaluation of functionality in patients with shoulder disorders. Dan Med J 2013;60:A4608.

9. Omar N, Alvi F, Srinivasan MS. An unusual presentation of whiplash injury: Long thoracic and spinal accessory nerve injury. Eur Spine J 2007;16 Suppl 3:275-7.

10. Gorski JM, Schwartz LH. Shoulder impingement presenting as neck pain. J Bone Joint Surg Am 2003;85-A:635-8.

11. Depalma AF. Surgical anatomy of acromioclavicular and sternoclavicular joints. Surg Clin North Am 1963;43:1541-50.

12. Salter EG Jr, Nasca RJ, Shelley BS. Anatomical observations on the acromioclavicular joint and supporting ligaments. Am J Sports Med 1987;15:199-206.

13. Levy O, Rath E. Traumatic soft tissue injuries of the shoulder girdle. Trauma 2002;4:223-35.

14. Wallace AW, Almedia I, Neumann L, Manning PA. Whiplash injuries to the shoulder-evaluation of a previously unrecognized problem. In: $9^{\text {th }}$ BESS Annual Meeting, Glasgow, Scotland, 14-15 May 1998.

15. Tossy JD, Mead NC, Sigmond HM. Acromioclavicular separations: Useful and practical classification for treatment. Clin Orthop Relat Res 1963;28:111-9.

16. Allman FLJr. Fractures and ligamentous injuries of the clavicle and its articulation. J Bone Joint Surg Am 1967;49:774-84.

17. Williams GR, Nguyen V, Rockwood CR. Classification and radiographic analysis of acromioclavicular dislocations. Appl Radiol 1989;Feb: 29-34.

18. Heers G, Götz J, Schubert T, Schachner H, Neumaier U, Grifka J, et al. MR imaging of the intraarticular disk of the acromioclavicular joint: A comparison with anatomical, histological and in-vivo findings. Skeletal Radiol 2007;36:23-8.

How to cite this article: Atoun E, Bano A, Tongel AV, Narvani A, Sforza G, Levy O. Acromioclavicular joint acceleration-deceleration injury as a cause of persistent shoulder pain: Outcome after arthroscopic resection. Indian J Orthop 2014;48:193-6.

Source of Support: Nil, Conflict of Interest: None.

\section{Staying in touch with the journal}

1) Table of Contents (TOC) email alert Receive an email alert containing the TOC when a new complete issue of the journal is made available online. To register for TOC alerts go to www.ijoonline.com/signup.asp.

\section{2) RSS feeds}

Really Simple Syndication (RSS) helps you to get alerts on new publication right on your desktop without going to the journal's website. You need a software (e.g. RSSReader, Feed Demon, FeedReader, My Yahoo!, NewsGator and NewzCrawler) to get advantage of this tool. RSS feeds can also be read through FireFox or Microsoft Outlook 2007. Once any of these small (and mostly free) software is installed, add www.ijoonline.com/rssfeed.asp as one of the feeds. 